Rafidain Journal of Science

Vol. 29, No.1, pp.73-85, 2020

DOI

https://rsci.mosuljournals.com

ISSN: 1608-9391
e-ISSN: 2664-2786

Received $9 / 3 / 2020$ Accepted 18/5/2020
تصميم وتصنيع هوائي شريحة رقيقة ثلاثي الحزمة مع تعزيز عرض الحزمة WiMAX ,WLAN, GSM الترددية لتطبيقات

$$
\begin{aligned}
& \text { "حمد عبد الكريم احمد")" } \\
& \text { قسم الفيزياء / كلية العلوم/ جامعة تكريت اهمبت اهمب } \\
& \text { فارس صالح عطالله } \\
& \text { قسم الفيزياء/ كية العلوم/ جامعة تكريت } \\
& \text { يسار عزالدين محمد علي }
\end{aligned}
$$$$
\text { قسم هندسة الكهرباء/ كلية الهندسة/ جامعة الموصل }
$$

*E-mail: A.A_Ahmed87@yahoo.com

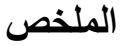

يتضمن هذا البحث تصميم نموذج لهوائي الثريحة الرقيقة ثلاثي الحزمة يناسب

تطبيقات الاتصالات اللاسلكية GSM و WLAN GiMAX الحجم الكلي للهوائي المقترح

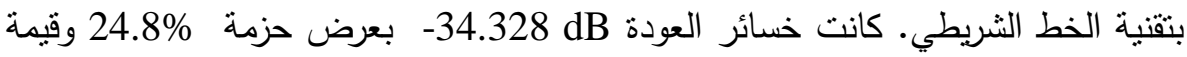
الكسب 2.8738 dB عند التردد 0.912 وخسائر العودة 37.375 dB بعرض حزمة 52.33\% وقيمة الكسب 2.04 dB عند التردد 1.842 GHz وخسائر العودة 27.753بعرض حزمة 40\% وقيمة الكسب 4.476 dB عند التزدد 2.4 وخسائر العودة 3.54 GHz 3.471 dB بعرض حزمة 4\% وقيمة الكسب dB 4.95 عند التزدد تم عرض نتائج المحاكاة باستخدام برنامج CST. نم تتفيذ الهوائي بتكلفة منخفضة وقد وجد تطابق مقبول بين النتائج العملية ونتائج المحاكاة لخسائر العودة.

الكلمات الدالة: هوائي الثريحة الرقبقة، متعدد الحزم، GSM، WWLAN ،WMAX ، .CST 


\section{المقدمة}

أصبح النظام اللاسلكي في الوقت الحالي جزءا مهما في حياتتا حيث ان معظم الاجهزة الكهربائية والالكترونية نستخدم

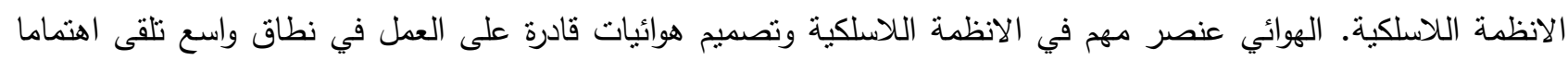

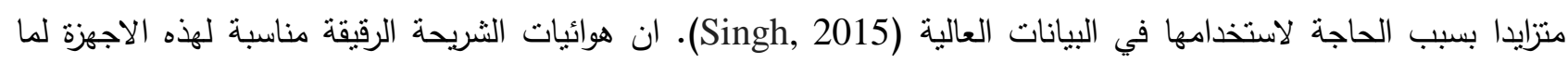

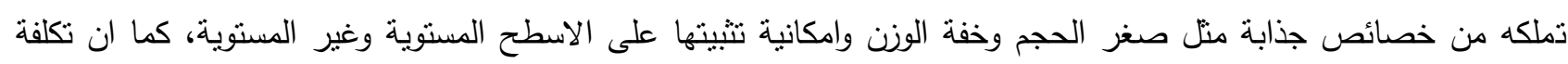

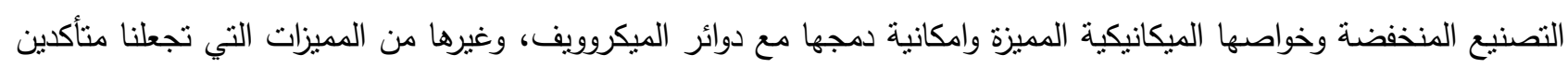

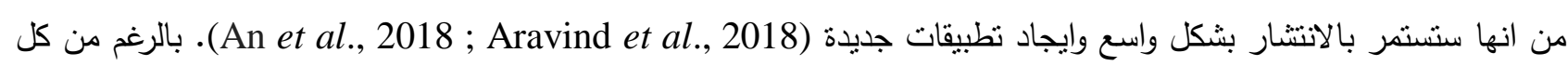
المميزات التي تم ذكرها الا ان هوائيات الثريحة الرقيقة لها بعض المساوئ اهمها ان لها عرض الهان حزمة ضيق وانخفاضا في الكسب

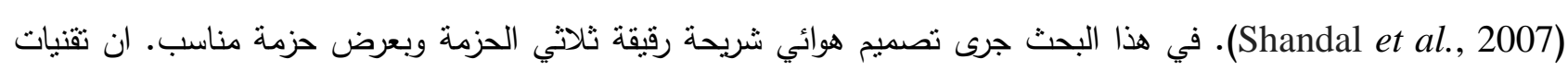

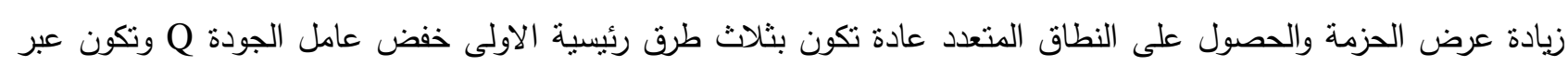

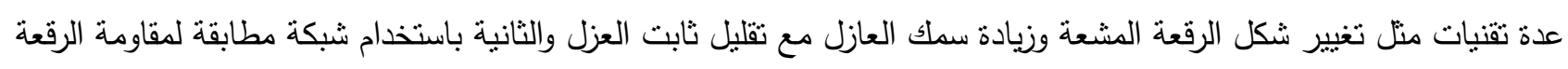
مع خط النقل عن طريق اضافة عنصر توليف او باستخدام الفتحات في الرقعة المشعة والثالثة عن طريق الرنين المتعدد وينم الحصول عليه عن طريق اضافة عناصر طفيلية (مستوية او مكدسة) (Hussain, 2018 ; Chen, 2018). ان الفائدة الرئيسة لاستخدام الهوائي متعدد الحزم هي في الاجهزة المحمولة، فبدلا من استخدام عدد من الهوائيات يمكن استخدام هوائي يناسب عدة

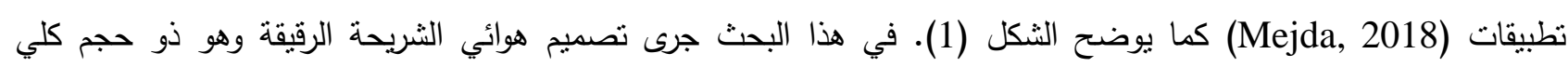
0.912 GHz مناسب تطبيقات (Huang, 2008) (GSM:1.805 -1.88 GHz) والثاني 1.842GHz يناسب تطبيقات (GSM:0.88 - 0.925GHz)

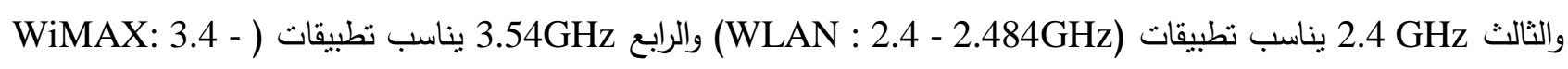
(Naji , 2018) (3.7GHz ) تمت تغذية الهوائي باستخدام تقنية الخط الثريطي بمقاومة 50د. نم تصميم الهوائي وعرض نتائج المحاكاة باستخدام برنامج CST.

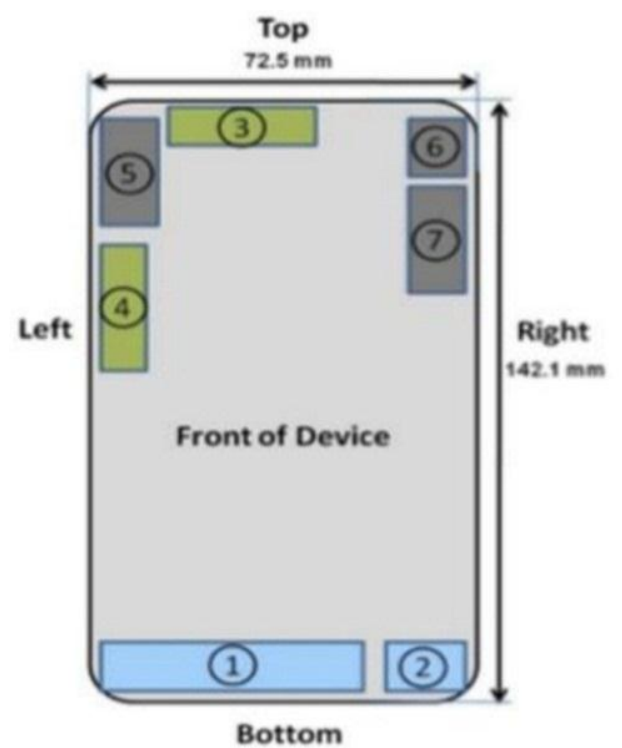

1. Main Antenna (Tx, Rx)

-CDMA/EVDO BCO/1/10

$-850 / 1900 \mathrm{MHz}$ GSM/GPRS/EDGE

-UMTS Band2/5

-LTE Band 25/26

2. LTE B41 Main Antenna (Tx, Rx)

3. BT/WLAN Antenna \#1 (Tx, Rx)

$-2.4 \mathrm{GHz}$ BT/WIFI

$-5 \mathrm{GHz}$ WIFI

4. WLAN Antenna \#2 (Tx, Rx)

$-2.4 \mathrm{GHz}$ WIFI

$-5 \mathrm{GHz}$ WIFI

5. MRD Antenna (Tx,Rx)

6. GPS Antenna (Rx only)

7. LTE B41 sub Antenna (Rx Only)

Bottom

الثكل 1: عدد ومواقع الهوائيات في هاتف Galaxy S5] 


\section{الجزء النظري}

يعتمد عمل الهوائي على عدد من الخصائص اهمها:

خسائر العودة Return losses تصف العلاقة بين القدرة الداخلة والمنعكسة لنظام يحتوي على شبكة مكونة من اثثين من الأطراف، عندما تكون قيمة خسائر العودة قلبلة يعني انه لخط النقل مواءمة عالية مع الهوائي وهذا يعني ان القدرة المنعكسة قليلة

وبالتالي عمل الهوائي يكون جيدا (Domènech , 2016). عرض الحزمة Band width نطاق التزددات الذي تظهر خلاله مميزات الهوائي وتكون عرض الحزمة مساوية للفرق بين التردد الاعلى والتردد الادنى المقابل لقيمة 10 dB- لخسائر العودة (Klopper , 2016).

B. $W=F_{H}-F_{L}$

$\mathrm{F}_{\mathrm{BW}}=\left(\frac{F_{H}-F_{L}}{F_{C}}\right) \times 100 \%$
وعرض الحزمة الكسرية تحسب بالعلاقة الاثية

الكسب Gain نسبة شدة الاشعاع الى شدة الاشعاع لهوائي Isotropic الذي يشع الطاقة بمقدار منساو في جميع الاتجاهات

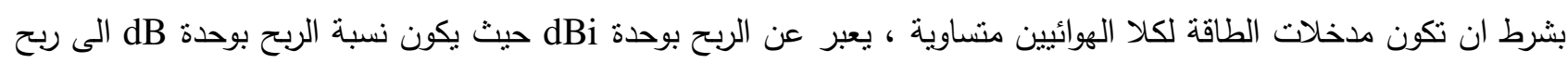
الهوائي الذي يشع في جميع الاتجاهات (Khan , 2016).

$\mathrm{G}=4 \pi \frac{\mathrm{U}\left(\theta_{i} \Phi\right)}{P_{\text {in }}}$

(Watt) Pin القدرة الداخلة

(Watt/unit) $\quad U(\theta, \Phi)$ شدة الاشعاع

نمط الاشعاع Radiation Pattern هو دالة رياضية او تمثيل شكلي لخواص اشعاع الهوائي كدالة للإحداثيات مع الزمن، تتضمن خواص الاشعاع كثافة القدرة المتدفقة وشدة الاشعاع وقوة المجال والاتجاهية والطور والاستقطاب، في معظم الحالات ينم ايجاد نمط الاشعاع في المنطقة البعيدة (Balanis , 2016). نسبة فولتية الموجة المنوقفة Voltage Standing Wave Ratio النسبة بين أعظم قيمة واقل قيمة لفولتية الموجة المتوقفة المتولدة في الخط نتيجة لحالة عدم المواعمة بالمقاومة عند طرف الحمل (Balanis, 2016). تصميم هوائي الثريحة الرقيقة التمهيدي تتم خطوات تصميم هوائي الثريحة الرقيقة عبر تطبيق مجموعة من المعادلات التجريبية لحساب الابعاد الاولية للرقعة المشعة التقليدية المستطيلة الثكل لهوائي الشريحة الرقيقة. (Rashid, 2018; Mishra, 2019) حساب عرض الرقعة المشعة (W) عند التردد 0.9 GHz وثابت العزل (ع) = 4.3 للهوائي وفق المعادلة (4): $\mathrm{W}=\frac{1}{2 \sqrt{\mu_{\mathrm{g}} \mathrm{g}}} \sqrt{\frac{2}{\mathrm{~g}_{\mathrm{r}}+1}}=\frac{c}{2 f_{\mathrm{g}}} \sqrt{\frac{2}{\mathrm{~g}_{\mathrm{r}}+1}}$

حيث ع عنثل السماحية النسبية للفراغ و 0 تمنل النفوذية المغناطيسية للفراغ و تمنل سرعة الضوء في الفراغ

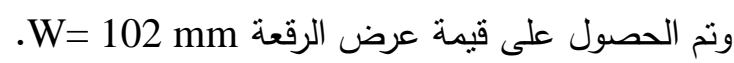

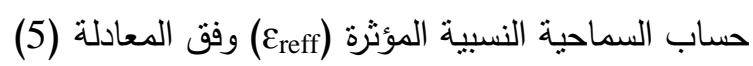


$\varepsilon_{\text {reff }}=\frac{\varepsilon_{r}+1}{2}+\frac{\varepsilon_{r}-1}{2}\left[1+12 \frac{\hbar}{w}\right]^{-1 / 2}$

(5)

ff $=\frac{c}{2 f \sqrt{\varepsilon_{\text {reff }}}}$

$\mathrm{L}=\mathrm{L}_{\mathrm{eff}}-2 \Delta \underline{\mathrm{L}}$

حساب الفرق بين الطول الكهربائي والطول الفيزيائي للرقعة المشعة (LL) وفق المعادلة (7)

وتم الحصول على قيمة السماحية النسبية المؤثرة

حساب الطول الكهربائي للرقعة المشعة (Leff) وفق المعادلة (6)

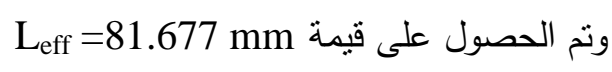

$\frac{\Delta L}{h}=0.412 \frac{\left(\varepsilon_{\text {reff }}+0.3\right)\left(\frac{W}{h}+0.264\right)}{\left(\varepsilon_{\text {reff }}-0.258\right)\left(\frac{W}{h}+0.8\right)}$

نم حسابها والحصول على القيمة.

حساب الطول الفيزيائي للرقعة (L) وفق المعادلة (8)

$$
\text { تم حسابه والحصول على القيمة. L }
$$
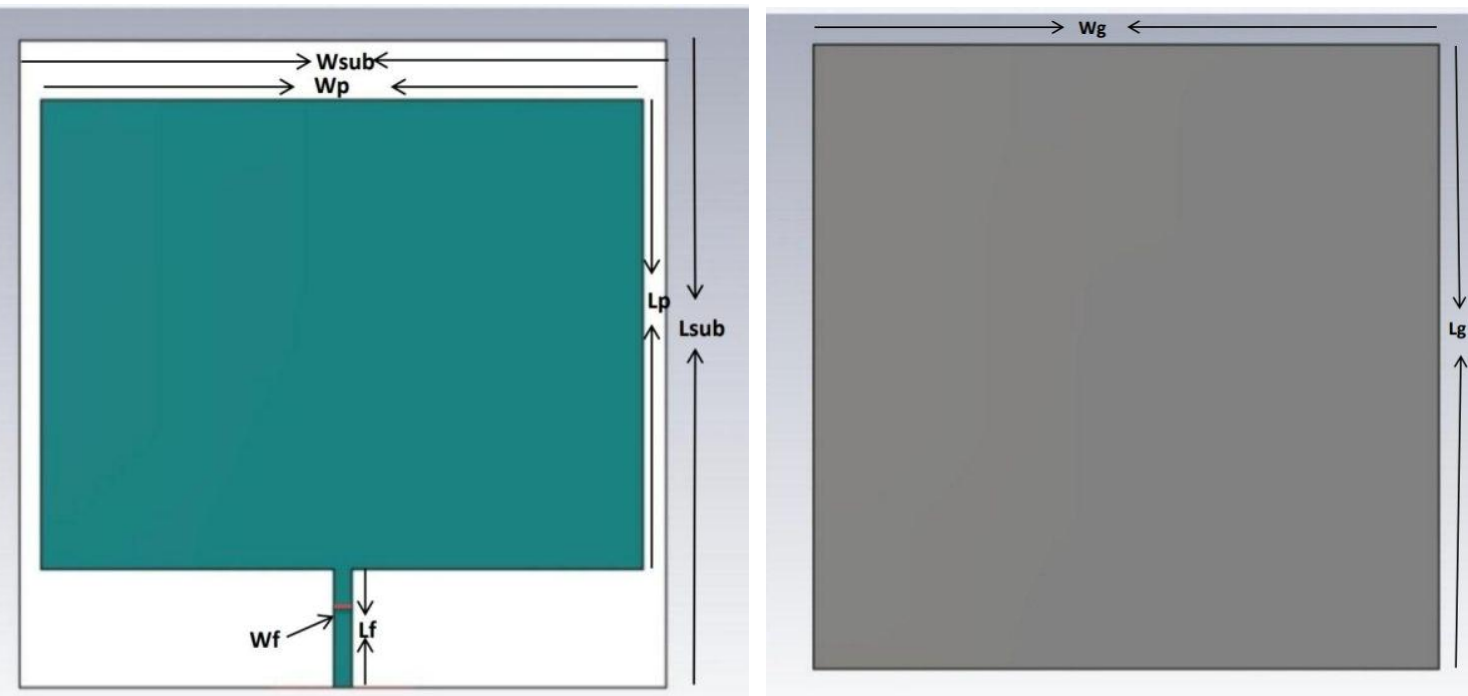

الرقعة

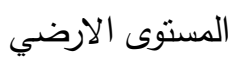

الثكل 2: الهوائي التمهيدي

الجدول 1: ابعاد الهوائي التمهيدي (جميع الابعاد مقاسة بوحدة الملي متر)

\begin{tabular}{|c|c|c|c|c|c|c|c|c|}
\hline Wg & Lg & Wf & Lf & Wp & Lp & Wsub & Lsub & البعدئ \\
\hline 110 & 110 & 3 & 20 & 102.3 & 80 & 110 & 110 & القيمة \\
\hline
\end{tabular}




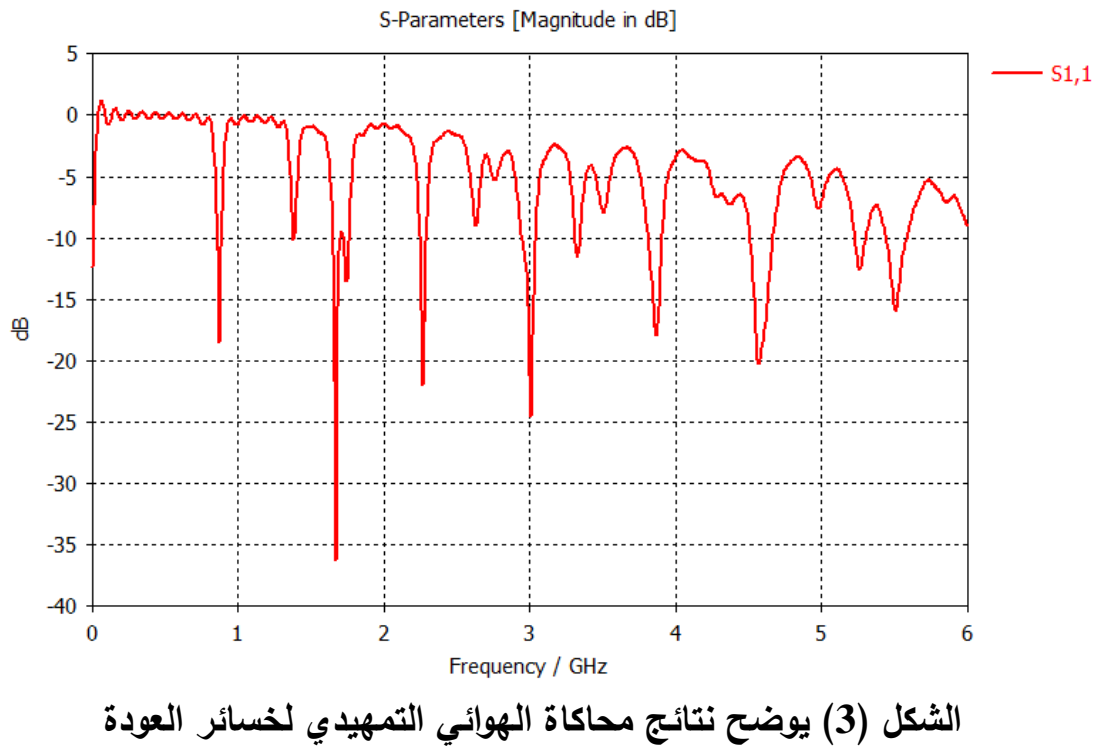

وقد تم تحسين الخواص الاشعاعية للهوائي باستخدام مجموعة من الخطوات حيث تم تقليل المستوى الارضي وقطع شكل خماسي من الرقعة وقطع منلثين من الزوايا السفلية للرقعة واضافة صفيحتين طفيليتين في المستوى الارضي كما في الثنكل (4) بهن وتم الوصول الى التصميم النهائي لهوائي الثريحة الرقيقة متعدد الحزم بأربعة ترددات 3.54 GHz و GHz

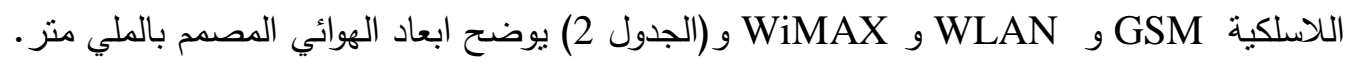
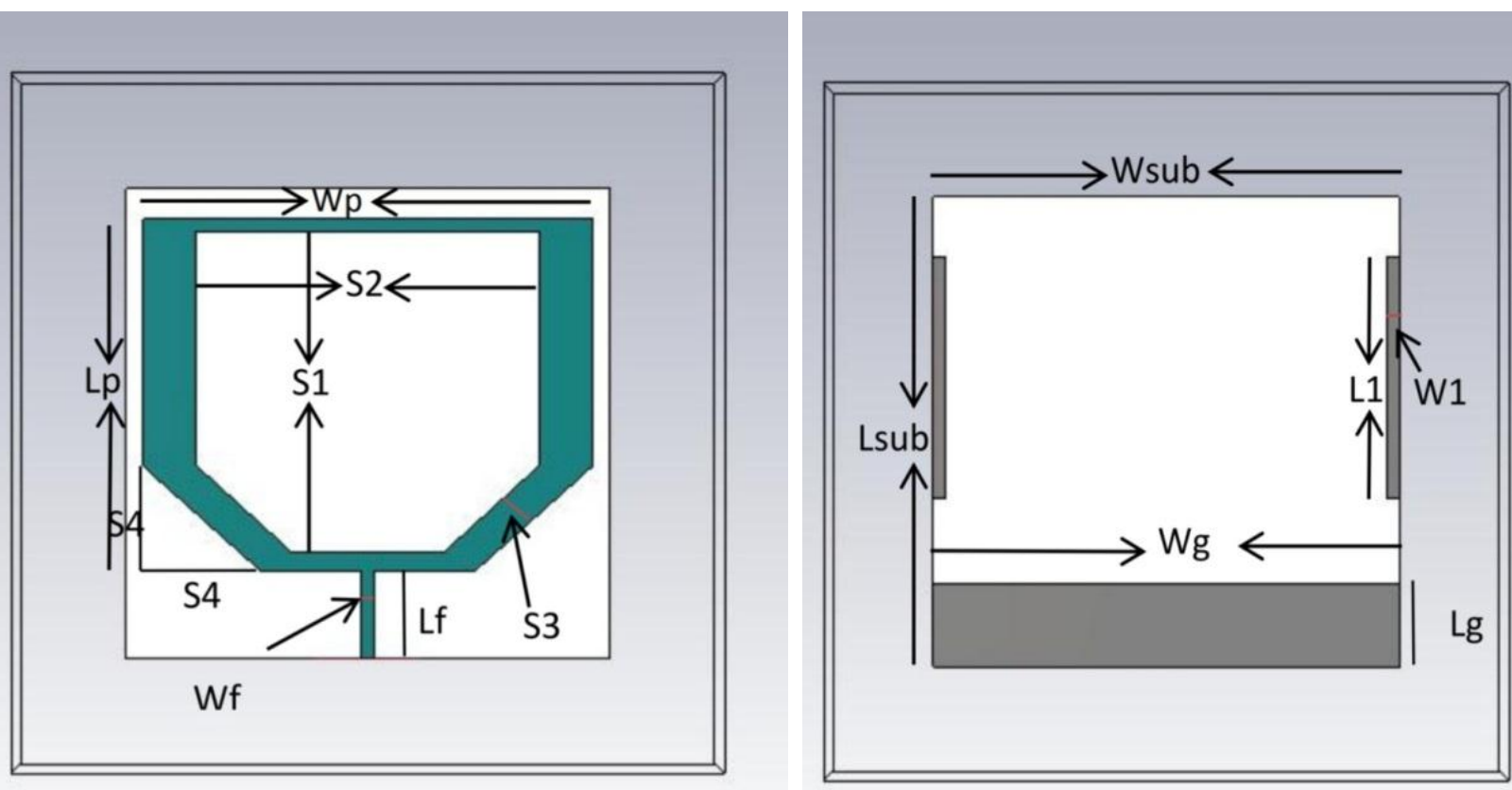

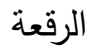
المستوى الارضي الثكل 4: الشكل النهائي للتصميم 
الجدول 2: ابعاد الشكل النهائي لتصميم (كل الابعاد مقاسة بوحدة الملي متر)

\begin{tabular}{|c|c|c|c|c|c|c|c|c|}
\hline Lf & Wf & Wp & Lp & Wg & Lg & Wsub & Lsub & البعد \\
\hline 20 & 3 & 102.3 & 80 & 110 & 19 & 110 & 107 & القيمة \\
\hline h & W1 & L1 & S5 & S4 & S3 & S2 & S1 & البعد \\
\hline 1.6 & 2 & 55 & 24.5 & 27.15 & 6 & 78.3 & 73 & القيمة \\
\hline
\end{tabular}

\section{النتائج والمناقشة}

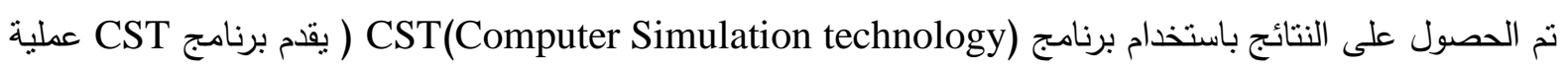
بسيطة في تكوين التراكيب بواجهة عرض امامية كما يوفر التحكم بتراكيب ثلاثية الابعاد ـ وبعد عملية بناء النموذج، تبدأ عملية المحاكاة وحساب النتائج. يستخدم برنامج CST تقنية Finite Integration Technique في التحليل العددي) وكانت النتائج :الثكل (5) يوضح رسم قيمة خسائر العودة كدالة مع التردد يمكن من خلالها تحديد ترددات العمل الاربعة 34.32 dB- عند

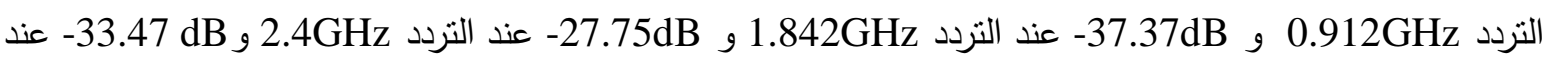

التزدد 3.54GHz

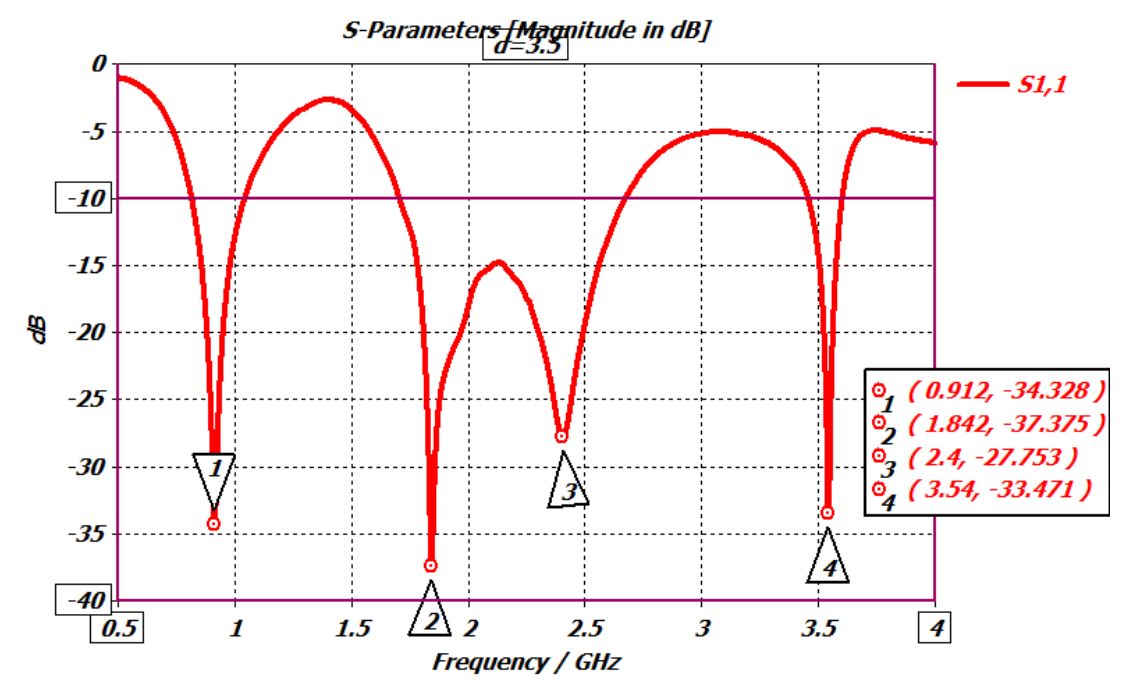

الثكل 5: خسائر العودة مع التردد

الثكل (6) يوضح مخطط سميث ويتضح من المخطط الجزء الحقيقي والخيالي لممانعة الهوائي المصمم ونلاحظ ان

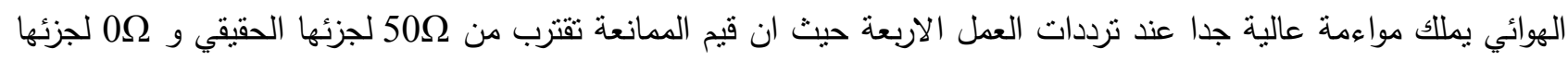

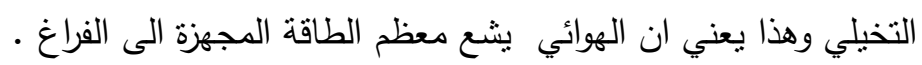




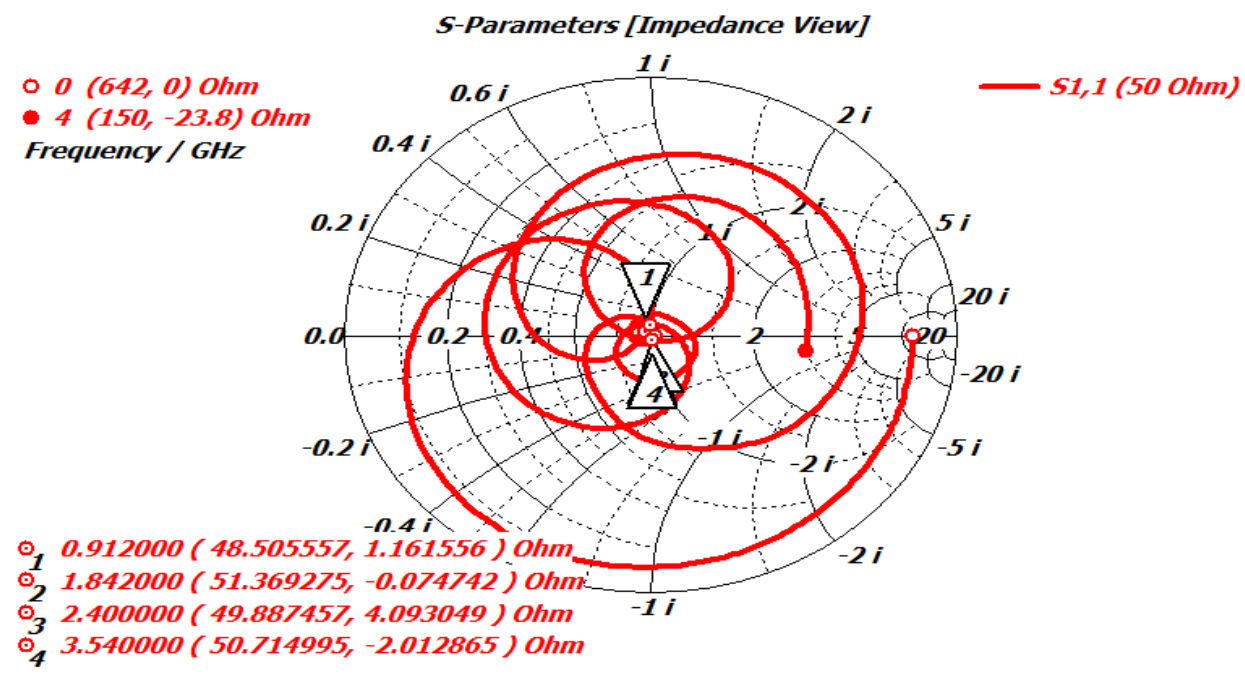

الشكل 6: مخطط سميث

الثكل (7) يوضح VSWR كدالة مع التردد وكما هو موضح في الثكل فان قيمتها عند ترددات العمل الاربعة ويتضح من الثكل انها تكون ذات قيم جيدة 1 > VSWR > 2 وانها تقترب من الواحد الذي يمنل القيمة المثالية لها لأنه عند هذه القيمة لا توجد

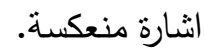

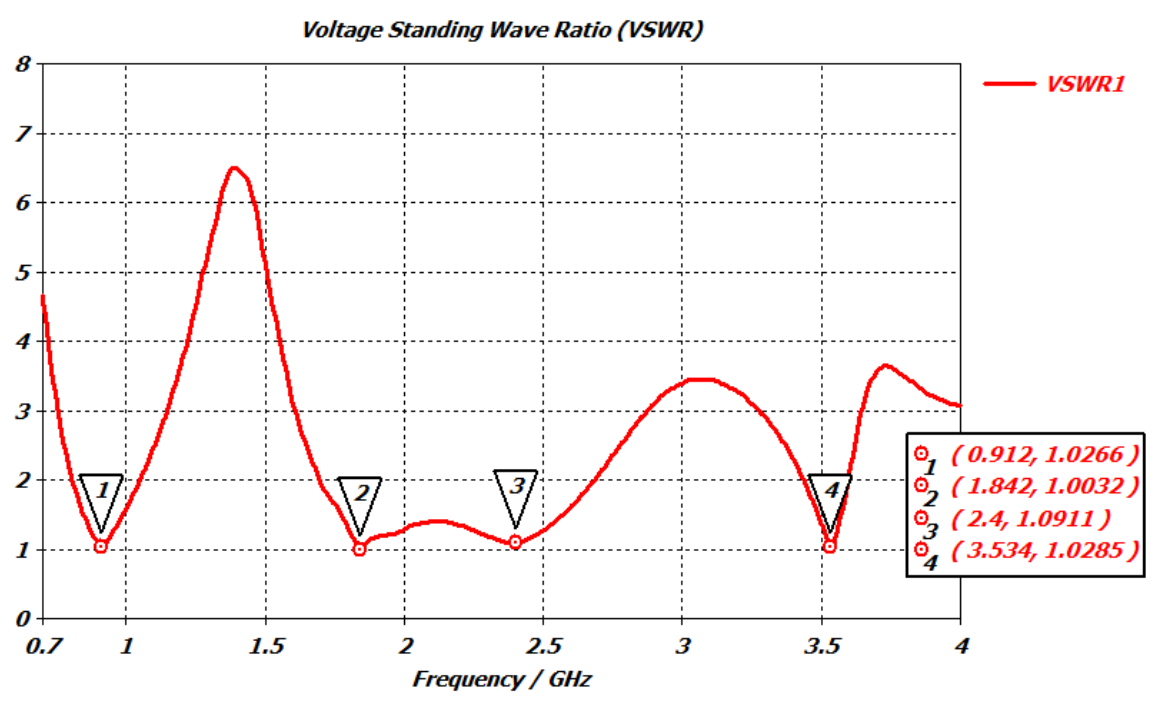

\section{الثكل 7: VSWR مع التردد.}

الثكل (8) يوضح رسم الجزء الحقيقي والجزء التخيلي للممانعة كدالة مع التزدد ويمكن ملاحظة حصول المواءمة العالية عند ترددات العمل الاربعة. 


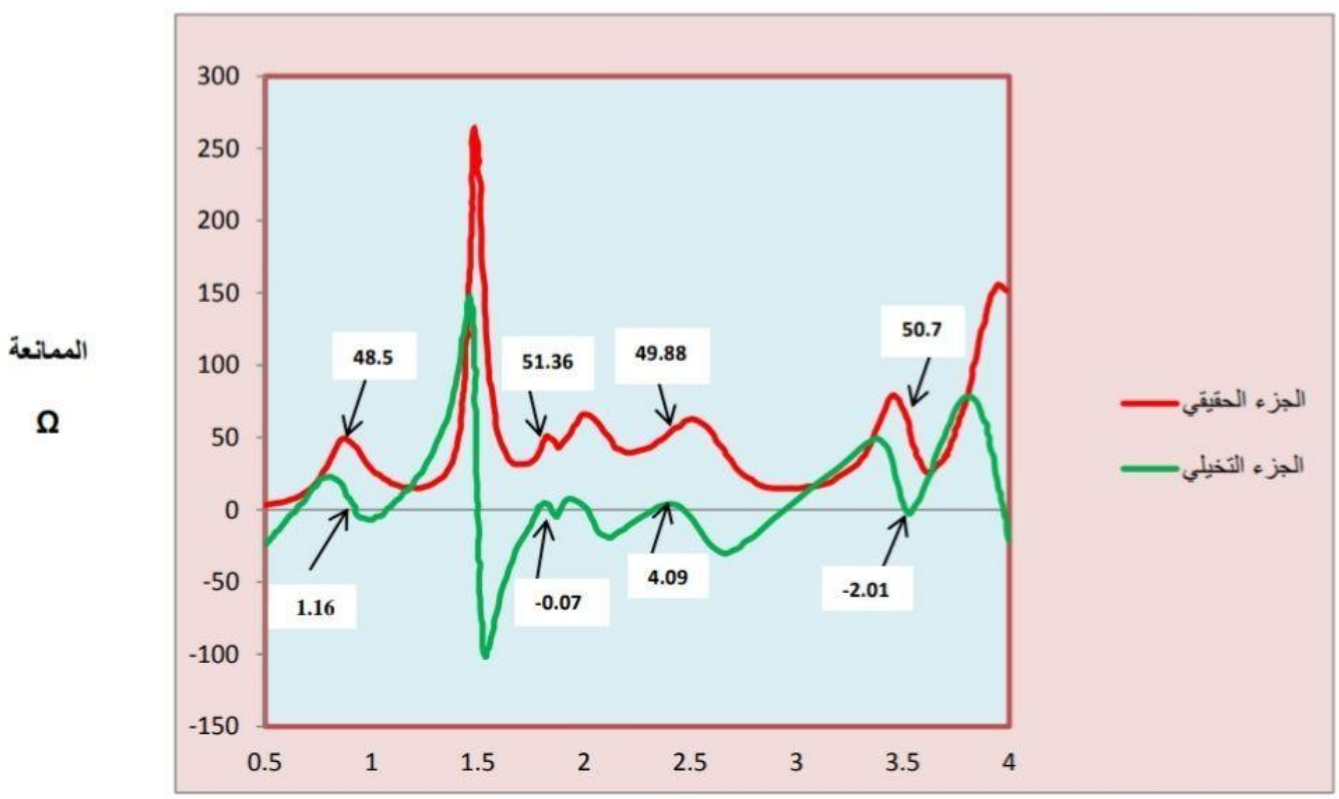

الشكل 8: يوضح المقاومة لكلا الجزأين الحقيقي والتخيلي مع التردد .

الثكل (9) كسب الهوائي بوحدة dB وكسب الهوائي بوحدة (dBi) كدالة مع التردد ويلاحظ ان الكسب يأخذ قيم موجبة عند ترددات العمل الاربعة و كانت قيمة الكسب 3.54GHz $6.84 \mathrm{GHz}$

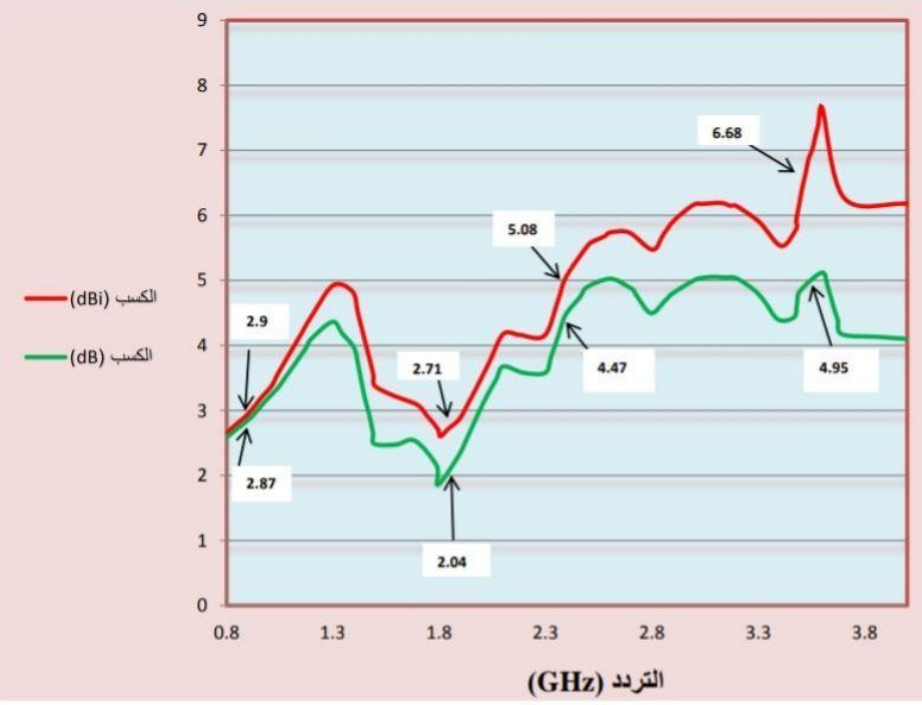

الشكل9: كسب الهوائي كدالة للتردد

الثكل (10) يوضح نمط اثعاع الهوائي عند ترددات العمل الاربعة ولكلا المستويين الكهربائي والمغناطيسي حيث يمنل المستوى

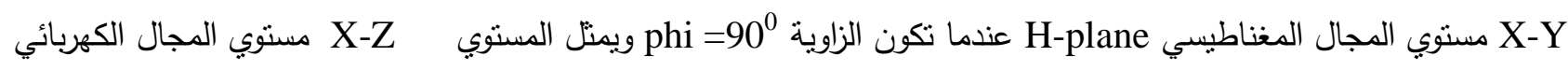
•phi = مستوي السمت عنما تكون الزاوية E-plane 


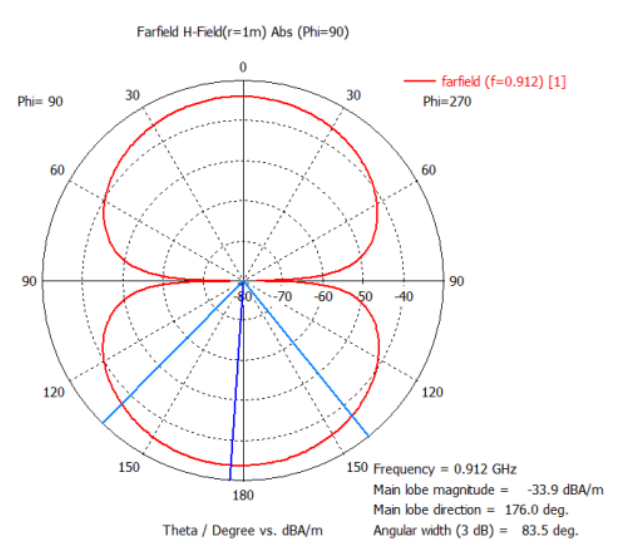

Farfield E-Field( $(r=1 \mathrm{~m})$ Abs (Phi $=0)$
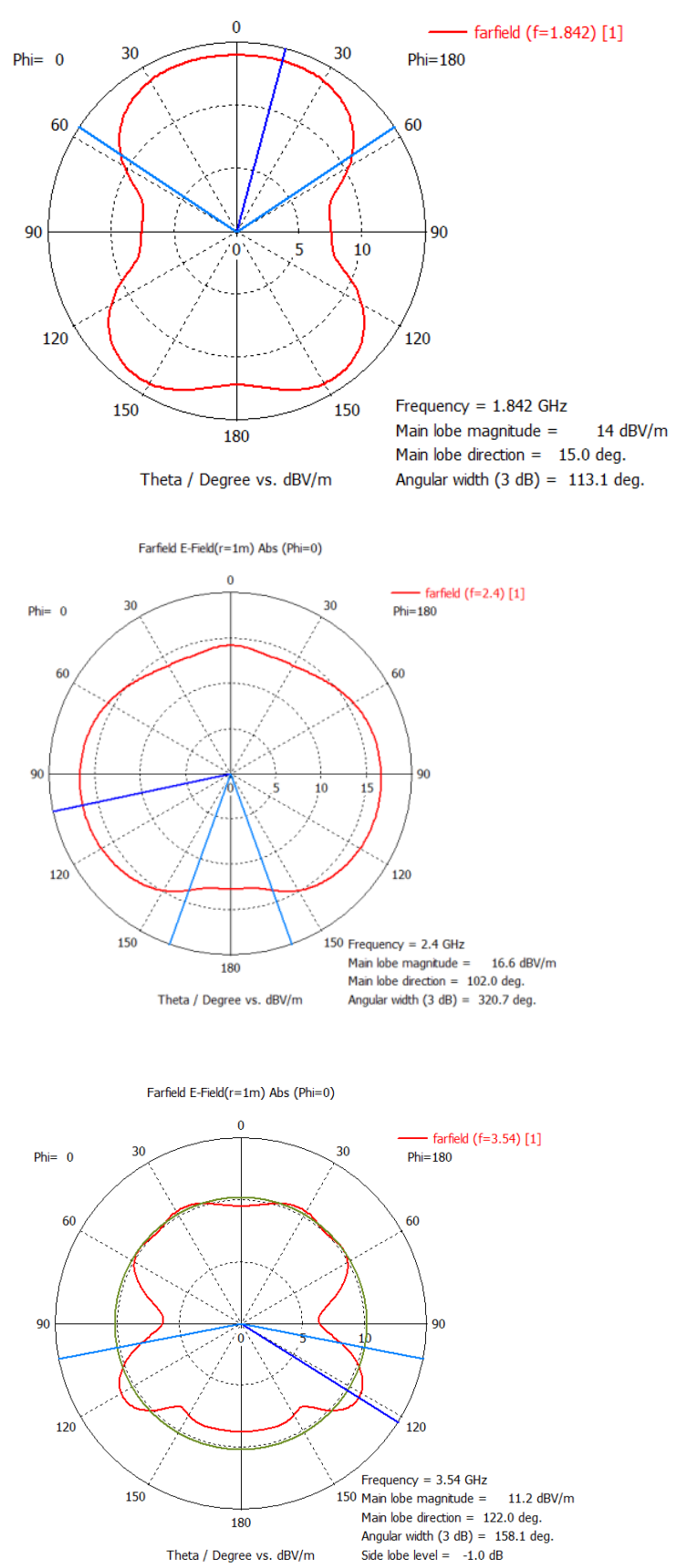
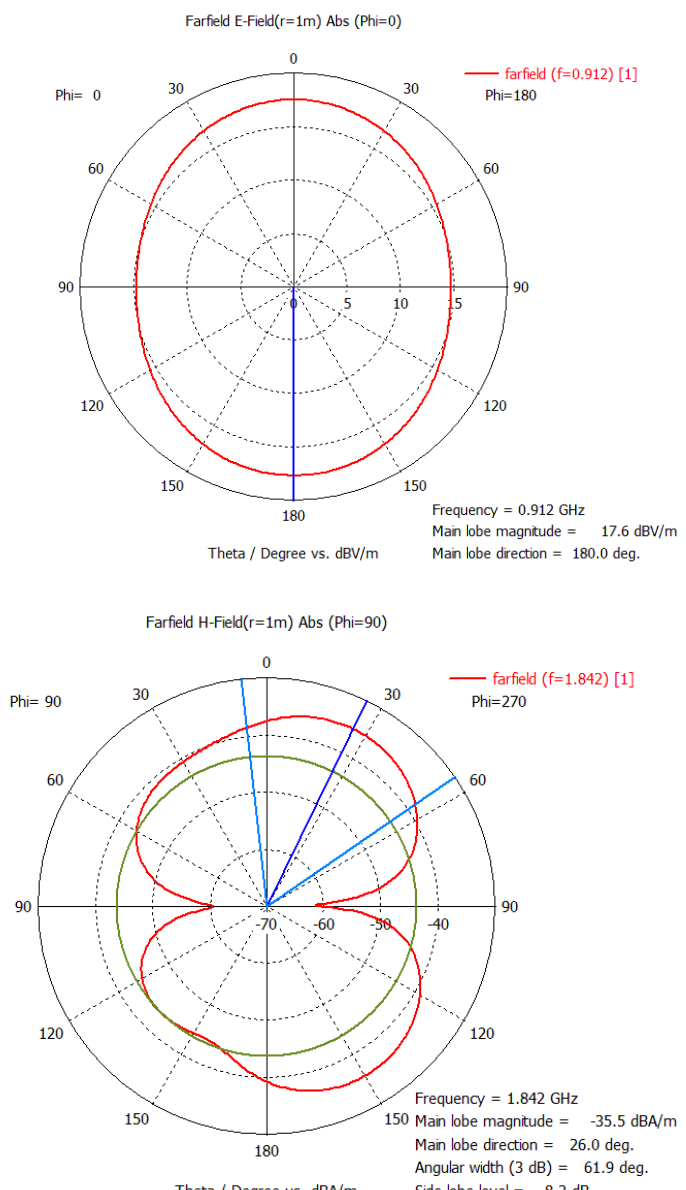

Theta / Degree vs. $\mathrm{dBA} / \mathrm{m} \quad$ Side lobe level $=-8.2 \mathrm{~dB}$

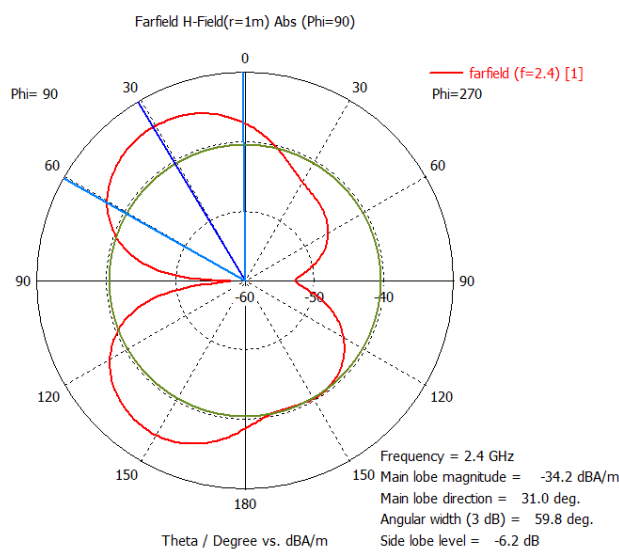

Farfield H-Field( $(\mathrm{r}=1 \mathrm{~m})$ Abs (Phi=90)

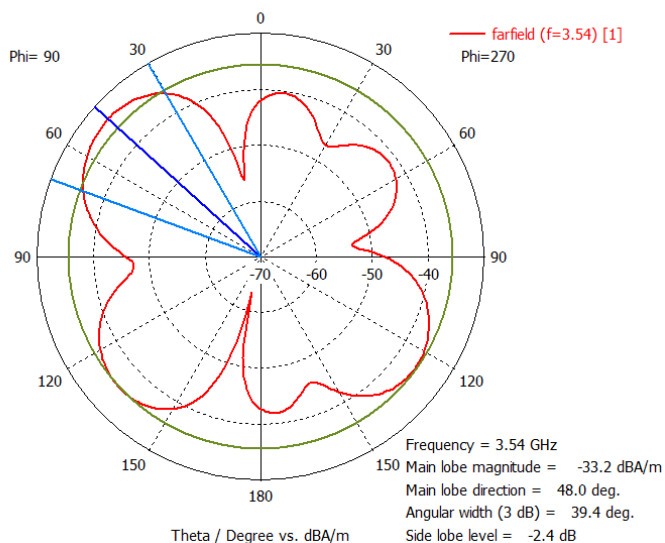


الثكل (11) يوضح توزيع التيار عند نرددات العمل الاربعة للهوائي المصمم وكما هو واضح بالثكل ان التبار يأخذ مسارا مختلفا عند كل نردد.
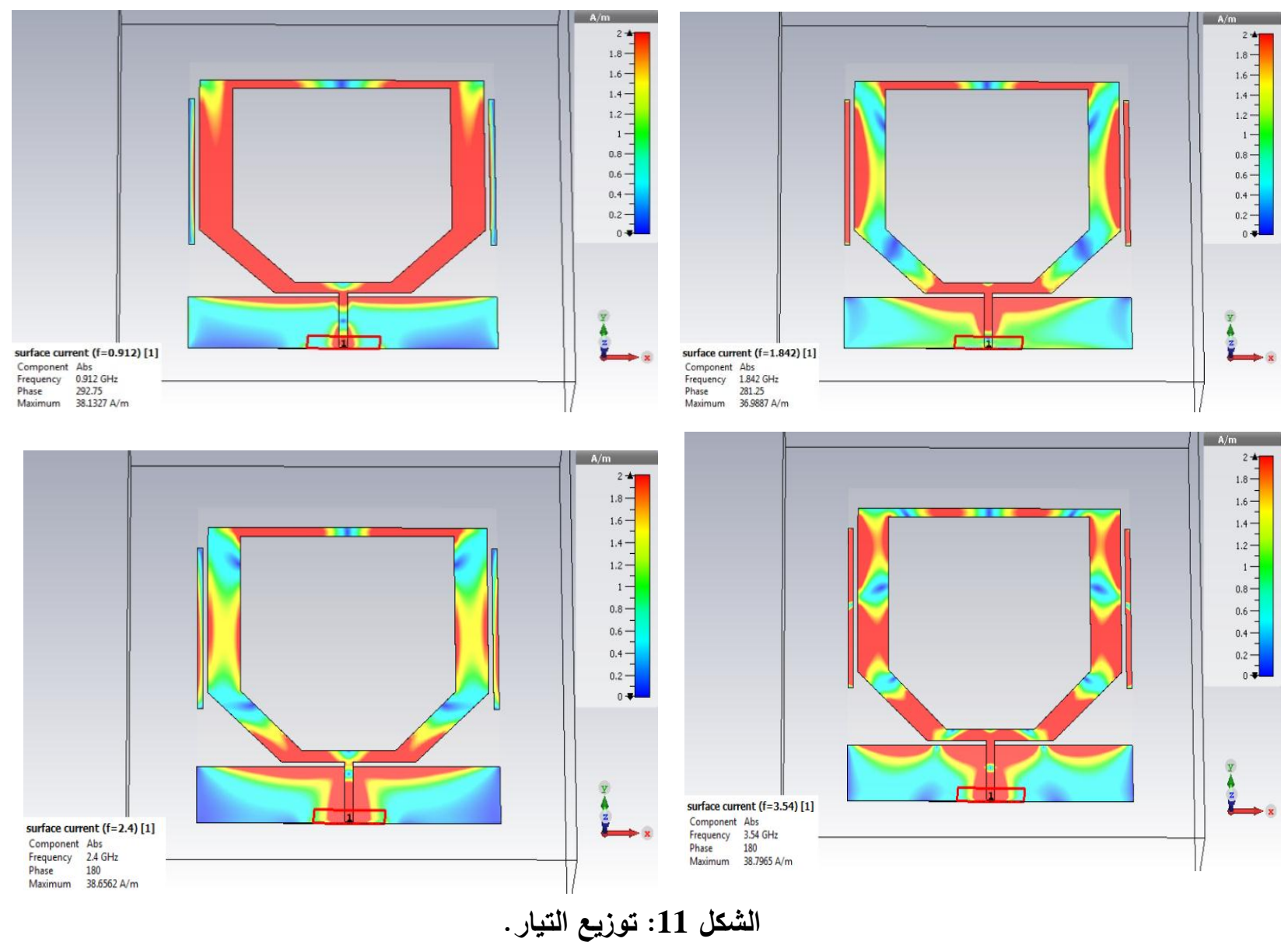

ويمكن تلخيص خصائص للنصميم المقترح بالجدول (3) الجدول 3: يوضح خصائص الهوائي

\begin{tabular}{|c|c|c|c|c|}
\hline الكسب & VSWR & خسائر العودة & التردد \\
\hline $24.8 \%$ & 2.8738 & 1.0392 & -34.328 & $0.912 \mathrm{GHz}$ \\
\hline $52.33 \%$ & 2.0411 & 1.0274 & -37.375 & $1.842 \mathrm{GHz}$ \\
\hline $40 \%$ & 4.4762 & 1.0854 & -27.753 & $2.4 \mathrm{GHz}$ \\
\hline $4 \%$ & 4.9543 & 1.0433 & -33.471 & $3.54 \mathrm{GHz}$ \\
\hline
\end{tabular}

\section{الجانب العملي}

تم تنفيذ الهوائي وقياس خسائر العودة في مختبر كلية هندسة الاكترونيات قسم الاتصالات جامعة نينوى بواسطة جهاز

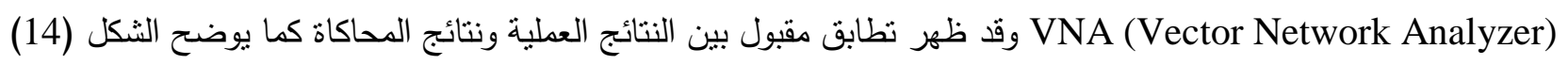



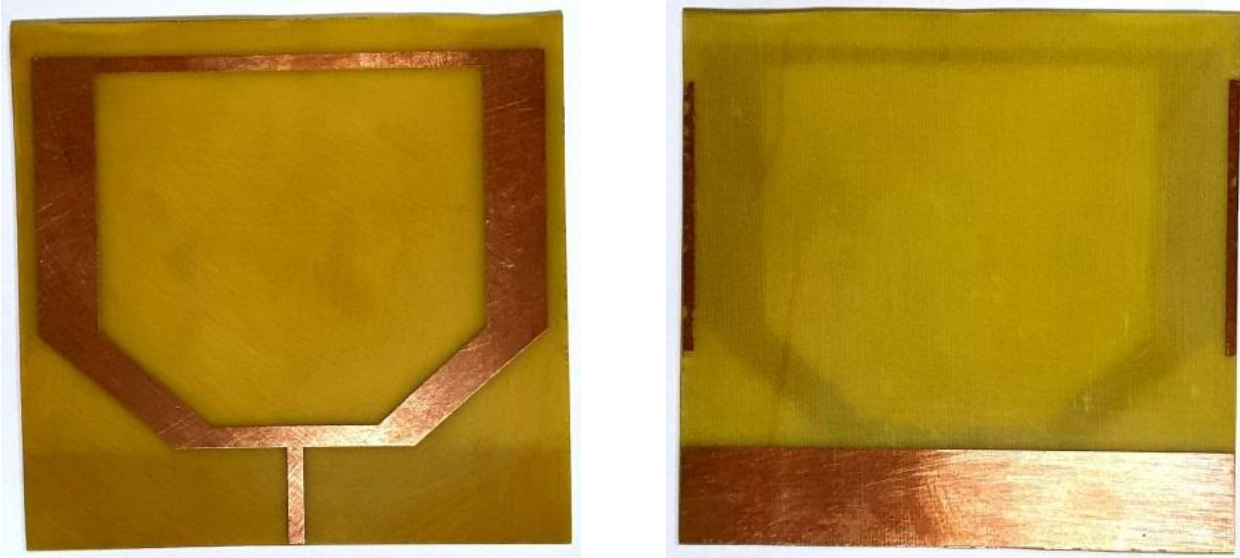

$$
\text { الشكل 12: الهوائي المطبوع }
$$

$$
\text { ويوضح الثكل (13) جهاز VNA نظهر عليه قيمة خسائر العودة للهوائي }
$$

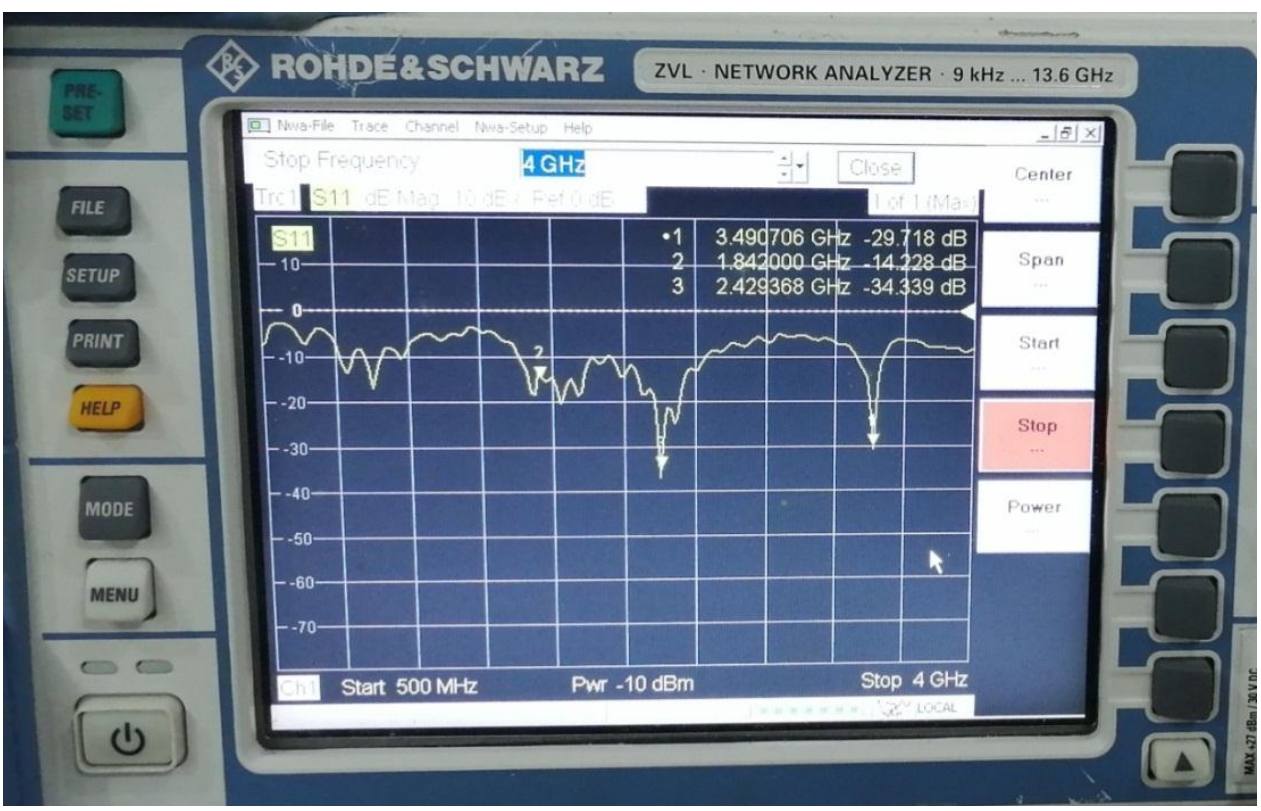

الشكل 13: القيمة العملية لخسائر العودة للهوائي

والثكل (14) مقارنة بين قيمة خسائر العودة للمحاكاة باستخدام برنامج CST والقيمة العملية لخسائر العودة باستخدام جهاز . VNA 


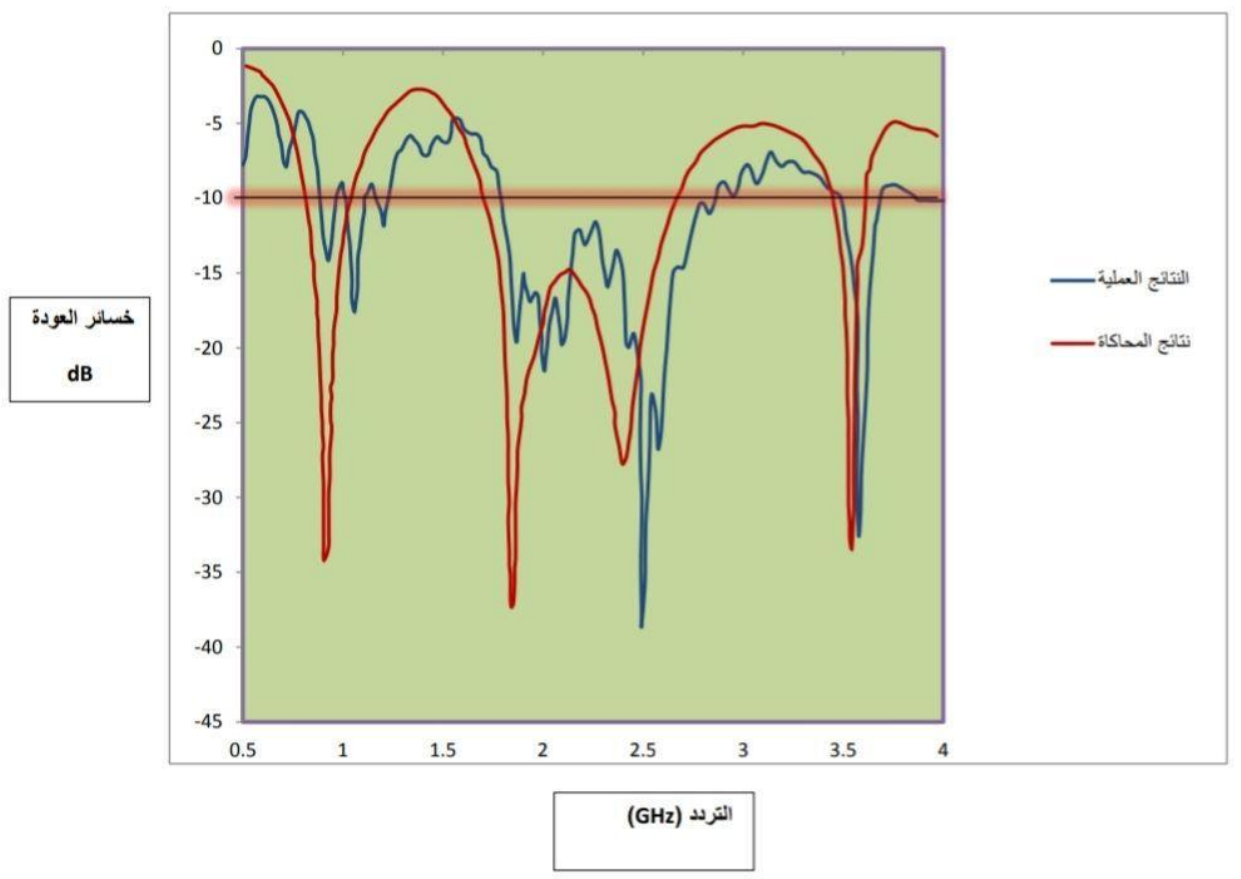

الثكل 14: القيمة العملية وقيمة المحاكاة لخسائر العودة

ويتضح من الثكل (14) ان هناك نوافقا مقبولا بين قيمة المحاكاة والقيمة العملية وان هناك اختلافا في القيمتين ويرجع ذلك الك الكات الى عدة اسباب اهمها ان القياسات لم تتم في مختبر عديم الصدى وان بعض هذه التزددات موجودة في جو المختبر بسبب وجود

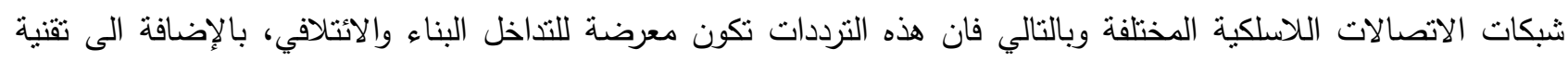
التصنيع البسيطة والتي تحتوي على نسبة خطا كزيادة او نقصان في ابعاد الرقعة او المستوى الارضي اون التهات العازل. الاستتتاجات

في هذا البحث تم تصميم هوائي شريحة رقيقة متعدد الحزم مناسب لتطبيقات WSM و WLAN و WiMAX

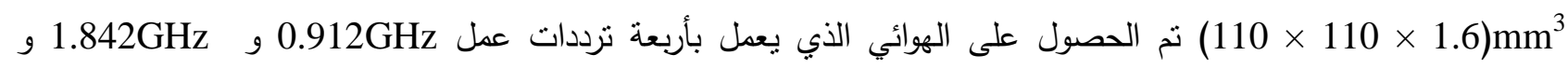
3.54GHz 2.4GHz باستخدام مجموعة من الاجراءات حيث تم قطع شكل خماسي من الرقعة وقطع منلثين من الزوايا السفلية

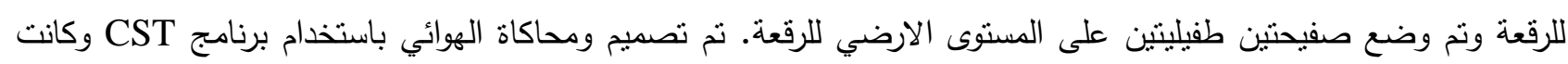
جميع خصائص الهوائي جيدة عند ترددات العمل الاربعة من حيث عرض الحزمة وخسارة العودة ونسبة فولتية الموجة المتوقفة

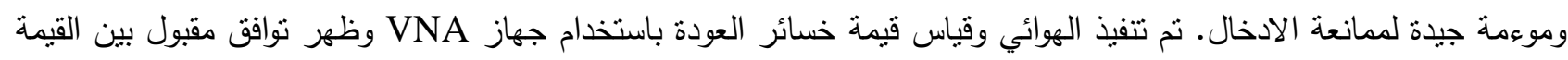
العملية وقيمة المحاكاة.

\section{المصادر}

Singh, Y.; Singh, D.; Gill, G. S. (2015). Design of wideband microstrip antenna for UWB applications. Int. Res. J. Eng. Technol, 2(5), 995-998.

An, W.; Li, Y.; Fu, H.; Ma, J.; Chen, W.; Feng, B. (2018). Low-profile and wideband microstrip antenna with stable gain for 5G wireless applications. IEEE Antennas and Wireless Propagation Letters, 17(4), 621-624.

Aravind, P.; Corlos, J.D.; lokesh, S.; Sridhar, B. (2018). Bandwidth improvement of microstrip patch antenna using parasitic patch. Int. J. Eme. Technol. in Com. Science and Ele., 25(5), 975-1335.

Shandal, S.A.; Mezaal, Y.S.; Mosleh, M.F.; Kadim, M. A. (2018). Miniaturized wideband microstrip antenna for recent wireless applications. Adv. Electromagnet., 7(5), 7-13. 
Hussain, H. S. (2016). Design and analysis of rectangular microstrip patch antenna operating at tm03 mode with single and stacked structure for bandwidth enhancement. Al-Nahrain J. for Eng. Sci., 19(2), 385-394.

Chen, Z.N.; Chia, M.Y.W. (2006). " Broadband Planar Antennas ". Hoboken eNJ NJ: John Wiley and Sons. pp. 978-0.

Mejda, Ms. G. (2018). Multiband Microstrip Patch Antenna for 4G(LTE). M. Sc. Thesis, Mohamed khider biskra university faculty of sciences and technology electrical engineering department, Algeria.

Huang, Y.; Boyle, K. (2008). " Antennas: from Theory to Practice ". John Wiley and Sons.

Naji, D. K. (2018). Design of compact dual- band and tri- band microstrip patch antennas. Int. J. Electromagnet Appl, 8(1), 26-34.

Domènech Ruiz, R. (2016). Design and analysis an array of Patch Antenna at $2.4 \mathrm{GHz}$ with circular polarization. Bachelor's thesis, Universität Politècnica de Catalunya.

Klopper, B. (2016). Fast design and optimization of one-dimensional microstrip patch antenna arrays. Doctoral dissertation, Stellenbosch: Stellenbosch University.

Khan, B. (2016). Adaptive vehicular antenna system for extended range cellular access. Center for Wireless Communications - Radio Technologies Research Unit, University of Oulu, Oulu Finland. Master's thesis.

Balanis, C. A. (2016). " Antenna Theory: Analysis and Design ". John Wiley and Sons.

Rashid, M.; Munir, M. E.; Khan, J.; Mahmood, K. (2018). Design of miniaturized multiband microstrip patch antenna using defected ground structure. Int. J. Adv. Comp. Sci. and App., 9(6), 168-173.

Mishra, R. (2019). Design and analysis of microstrip patch antenna for wireless communication. Int. J. Innovat. Tec. and Exploring Eng., 8(7), 2278-3075.

\title{
Design and Implementation of Tri Band Microstrip Patch Antenna with Bandwidth Enhancement for GSM, WLAN, WiMAX System
}

\author{
Ahmed A. Ahmed \\ Department of Physics/ College of Science/ University of Tikrit \\ Fares S. Alatallah \\ Department of physics/ College of Sciencel University of Tikrit \\ Yessar. E. Mohammed Ali \\ Department of Electrical Engineering/ Engineering College/ University of Mosul
}

\begin{abstract}
In this research design of tri-band microstrip antenna suitable for wireless GSM, WLAN and WiMAX application. The total size of the proposed antenna $(107 \times 110 \times 1.6) \mathrm{mm}^{3}$. The substrate material type is FR-4(epoxy). Return losses of $-34.328 \mathrm{~dB}$, bandwidth $24.8 \%$, gain $2.8738 \mathrm{~dB}$ at frequency $0.912 \mathrm{GHz}$ and return losses $-37.375 \mathrm{~dB}$, bandwidth $52.33 \%$, gain $2.0411 \mathrm{~dB}$ at frequency $1.842 \mathrm{GHz}$ and return losses $-27.753 \mathrm{~dB}$, bandwidth $40 \%$, gain $4.4762 \mathrm{~dB}$ at frequency $2.4 \mathrm{GHz}$ and return losses $-33.471 \mathrm{~dB}$, bandwidth $4 \%$, gain $4.9543 \mathrm{~dB}$ at frequency $3.534 \mathrm{GHz}$. The simulated results are obtained using the software computer simulation technology CST. The simulated results are verified with experimental results which are in acceptable agreement.
\end{abstract}

Keywords: Multiband, Microstrip antenna, GSM, WLAN, WiMAX. 